\title{
A DISCRETE ANALOG OF INVERTED TOPP-LEONE DISTRIBUTION: PROPERTIES, ESTIMATION AND APPLICATIONS
}

\section{AHMED SEDKY ELDEEB ${ }^{1,2,}$, MUHAMMAD AHSAN-UL-HAQ ${ }^{3,4}$, AYESHA BABAR $^{5}$}

1Department of Business Administration, College of Business, King Khaled University, Saudi Arabia

2 Department of Statistics, Mathematics and Insurance, Alexandria University, Egypt

${ }^{3}$ College of Statistical \& Actuarial Sciences, University of the Punjab, Pakistan

${ }^{4}$ Quality Enhancement Cell, National College of Arts, Lahore Pakistan

${ }^{5}$ School of Statistics, Minhaj University Lahore Pakistan

*Corresponding author: asedky@kku.edu.sa

ABSTRACT. In this study, a discrete inverted Topp-Leone (DITL) distribution is proposed by utilizing the survival discretization approach. The proposed distribution's mathematical features were derived. The maximum likelihood (ML), method of least squares (LS), weighted least squares (WLS), and Cramer Von-Mises (CVM) estimation techniques were used to estimate the parameter. The theoretical results of the ML, LS, WLS, and CVM estimators were demonstrated via a comprehensive simulation study. The proposed DITL distribution has been applied to analyze two count data sets number of deaths due to Covid-19 in Pakistan and India and the findings show the relevance of the proposed distribution.

\section{INTRODUCTION}

In December 2019, the first incidence of COVID-19 was reported in the Chinese city of Wuhan. COVID-19 is an extremely contagious disease. In Pakistan, the first case was reported on February 26, 2020 [1]. The first death was reported in Pakistan on March 20, 2020.

Received June 2nd, 2021; accepted July 8th, 2021; published August 2nd, 2021.

2010 Mathematics Subject Classification. 62E10.

Key words and phrases. discretization; inverted Topp-Leone distribution; moments; count data; data analysis.

C2021 Authors retain the copyrights of their papers, and all open access articles are distributed under the terms of the Creative Commons Attribution License. 
Many researchers make efforts to study the patterns of pandemic Covid-19 and provide models which better fit the data and can be used to have an idea about the expected number of cases to help the government to take decisions regarding precautionary measures. These efforts include the derivation of different probabilistic models and time series modeling of the data such as a new discrete Lindley [2], discrete Marshall-Olkin generalized exponential distribution to model the daily new cases in Egypt [3], a new discrete generalized distribution to analyze the count of daily cases in Hong Kong and Iran [4], a mathematical model known as SIR is used to predict the daily new cases in China [5,6], the logistic growth model is used to estimate the final size and its peak time of coronavirus epidemic [7], autoregressive time series model is used to forecast the recovered and confirmed cases [8], and discrete Marshall-Olkin Lomax distribution is used to estimate the daily new cases in Australia [9].

Practically, lifetime data sets are often recorded as whole numbers of counts. To model the count data, there are few classical distributions as geometric, Poisson, negative binomial, etc. these models sometimes do not provide a better fit due to the complex behavior of data. From the last few decades, researchers have paid attention to introduced discrete type distributions which meet the required need to model the complex behavior of data sets. Several discretization approaches are available in the literature. A detailed systematic review was conducted on discretization approaches [10]. Among all approaches, one of the most important is the survival discretizing approach due to its important feature of keeping the original form of the survival function.

"Let $X$ a continuous random variable. If $X$ has a survival function $S_{X}(x)$, then the discrete random Variable $Y=[X]$, where $[X]$ indicates the smallest integer part or equal to $X$, have probability mass function (PMF) written as":

$$
P(Y=k)=\sum_{i=0}^{1}(-1)^{i} S_{X}(k+i) \cdot k=0,1,2,3, \ldots
$$

Using survival discretization approach authors have derived discrete distributions. Some of these include discrete Weibull [11], discrete Rayleigh [12], discrete Burr and Pareto [13], discrete inverse Weibull [14], discrete Lindley [15], discrete generalized Rayleigh [16], discrete Bilal [17], discrete Nadarajah-Haghighi [18], discrete Burr-Hutke [19] and many others. 
The inverted Topp-Leone distribution [20] was derived for the analysis of reliability observations. A comprehensive discussion about its mathematical properties, reliability characteristics, stochastic ordering, and parameter estimation via complete and censored samples, among others is also presented in the mentioned paper. The corresponding survival function is given by

$$
S(x)=\frac{(1+2 x)^{\delta}}{(1+x)^{2 \delta}}, x>0, \delta>0
$$

where $\delta$ is the shape parameter.

The goal of this study is to introduce a new one-parameter discrete model called the discrete inverted Topp-Leone (DITL) distribution, which is based on the survival function approach of discretization. The DITL distribution can be used to model the over-dispersed data sets. We derive some of its properties such as, survival and hazard function, quantile function, moments, and generating function. The maximum likelihood, Cramer-von Mises, least-square, and weighted least square estimation methods are used to estimate the model's parameter. A simulation study is conducted to elaborate on the performance of these estimation methods. In the end, we will use data sets about the number of deaths due to coronavirus in Pakistan and India to illustrate the importance of the proposed distribution.

The organization of the article is as follows. Section 2 contains the derivation of the proposed distribution and its features. Section 3 addressed maximum likelihood estimation, as well as the least-squares, weighted least squares, and Cramer von Mises approach. In Section 4, a complete simulation study is used to assess the behavior of these estimators. The proposed distribution's application is discussed in Section 5. The conclusion has been presented in the final section.

\section{THE DITL DISTRIBUTION AND PROPERTIES}

Using a discretization approach based on the survival function, the discrete inverted Topp-Leone distribution has been developed. The probability mass function (pmf) of DITL distribution can be represented as

$$
P(x)=\frac{(1+2 x)^{\delta}}{(1+x)^{2 \delta}}-\frac{(3+2 x)^{\delta}}{(2+x)^{2 \delta}}, \quad \delta>0, \quad x=0,1,2, \ldots
$$


The pmf plots of the DITL distribution with some selected values of parameter $\delta$ are presented in Figure 1.
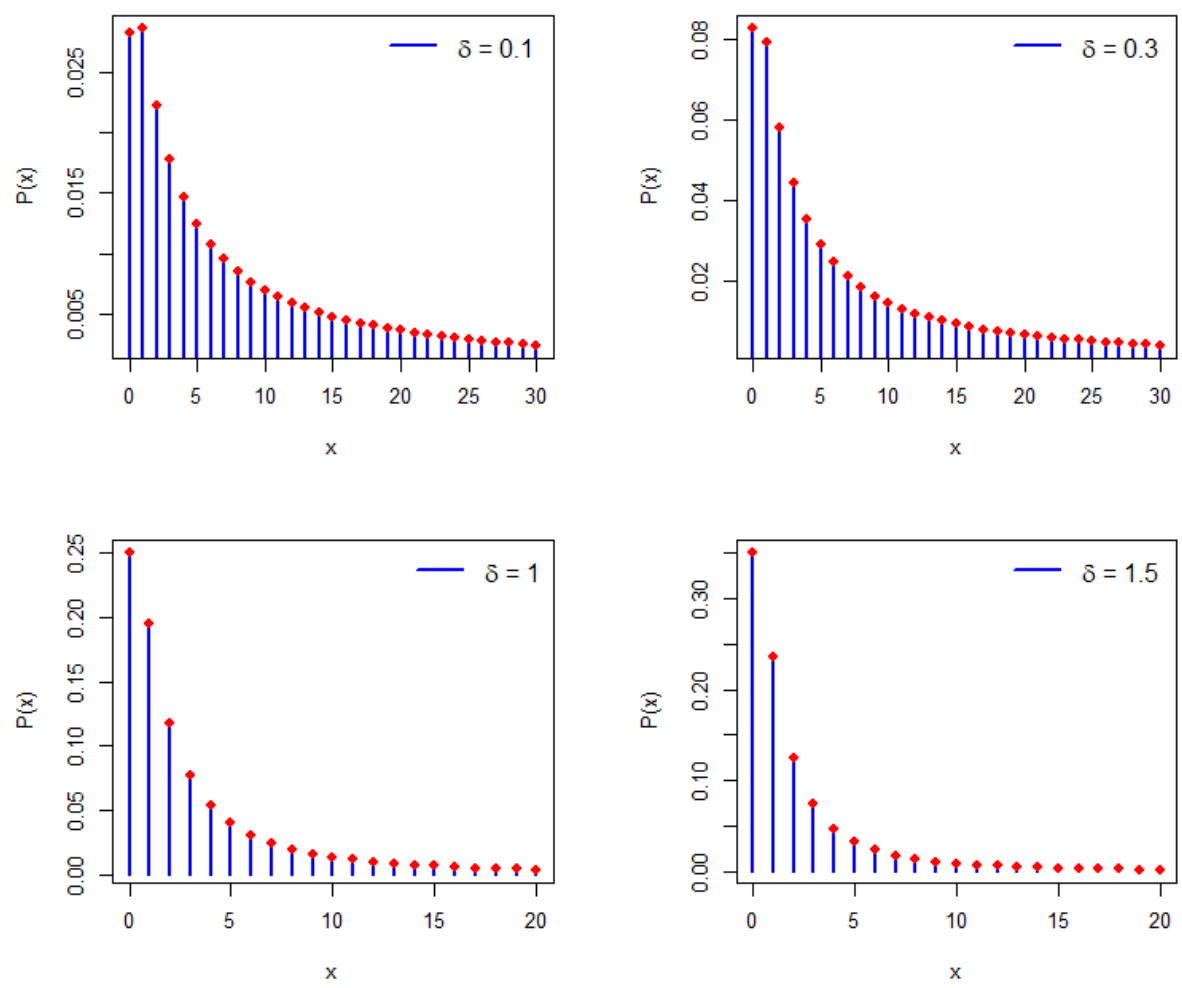

Figure 1: Behavior of pmf of DITLD for different parameter values

The cumulative distribution and survival functions of DITL are given, respectively as

$$
F(x)=1-\frac{(3+2 x)^{\delta}}{(2+x)^{2 \delta}}
$$

and,

$$
S(x)=\frac{(3+2 x)^{\delta}}{(2+x)^{2 \delta}}
$$

The hazard function of the DITL distribution is obtained using Eq. (3) and Eq. (5). The behavior of the hazard function is illustrated in Figure 2.

$$
h(x)=\frac{\frac{(1+2 x)^{\delta}}{(1+x)^{2 \delta}}-\frac{(3+2 x)^{\delta}}{(2+x)^{2 \delta}}}{\frac{(3+2 x)^{\delta}}{(2+x)^{2 \delta}}}
$$


or

$$
h(x)=\frac{(1+2 x)^{\delta}(2+x)^{2 \delta}}{(1+x)^{2 \delta}(3+2 x)^{\delta}}-1
$$

where $\delta>0 \& x=0,1,2, \ldots$. Note that, for $x \rightarrow 0$ the HRF turn into

$$
h(0)=\left[\left(\frac{4}{3}\right)^{\delta}-1\right]
$$
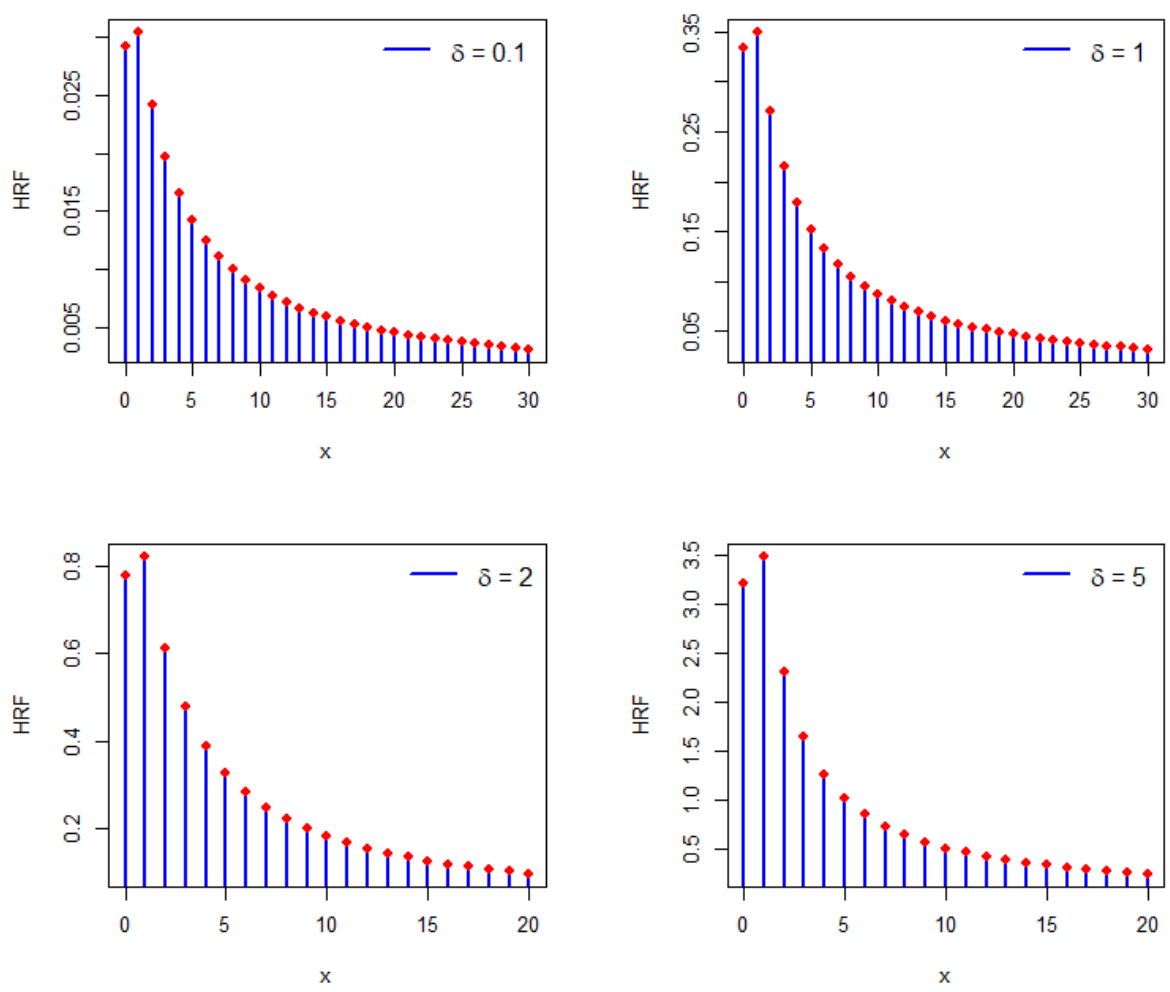

Figure 2: Behavior of HRF of DITLD for different parameter values

The reverse hazard function of DITL is given as

$$
r^{*}(x)=\frac{P(x)}{F(x)}=\frac{(1+2 x)^{\delta}(2+x)^{2 \delta}-(3+2 x)^{\delta}(1+x)^{2 \delta}}{(1+x)^{2 \delta}\left[(2+x)^{2 \delta}-(3+2 x)^{\delta}\right]}
$$

The second rate of failure of DITL is

$$
\mathrm{r}^{* *}(\mathrm{x})=\log \left[\frac{S(x)}{S(x+1)}\right]=\log \left[\frac{(3+2 x)^{\delta}(3+x)^{2 \delta}}{(2+x)^{2 \delta}(5+2 x)^{\delta}}\right]
$$

The recurrence relation for generating the probabilities of discrete DITL distribution is given by 


$$
\frac{P(x+1)}{P(x)}=\frac{(1+x)^{2 \delta}(2+x)^{2 \delta}\left[(3+2 x)^{\delta}(3+x)^{2 \delta}-(5+2 x)^{\delta}(2+x)^{2 \delta}\right]}{(2+x)^{2 \delta}(3+x)^{2 \delta}\left[(1+2 x)^{\delta}(2+x)^{2 \delta}-(3+2 x)^{\delta}(1+x)^{2 \delta}\right]}
$$

or

$$
P(x+1)=\frac{(1+x)^{2 \delta}\left[(3+2 x)^{\delta}(3+x)^{2 \delta}-(5+2 x)^{\delta}(2+x)^{2 \delta}\right]}{(3+x)^{2 \delta}\left[(1+2 x)^{\delta}(2+x)^{2 \delta}-(3+2 x)^{\delta}(1+x)^{2 \delta}\right]} P(x) .
$$

\subsection{Quantile Function}

The $\mathrm{p}^{\text {th }}$ quantile function of DITL distribution is given by

$$
x_{p}=\frac{\sqrt{4\left((1-p)^{(1 / \delta)}-1\right)^{2}-4(1-p)^{(1 / \delta)}\left((1-p)^{1 / \delta}-1\right)}-2\left((1-p)^{1 / \delta}-1\right)}{2(1-p)^{(1 / \delta)}} .
$$

\subsection{The moments of DITL distribution}

The non-central moments of DITL distribution can be obtained using Eq. (3) as follows:

$$
\begin{array}{r}
\mu_{r}^{\prime}=\sum_{x=0}^{\infty} x^{r} P(x) \\
\mu_{r}^{\prime}=\sum_{x=0}^{\infty} x^{r}\left[\frac{(1+2 x)^{\delta}}{(1+x)^{2 \delta}}-\frac{(3+2 x)^{\delta}}{(2+x)^{2 \delta}}\right]
\end{array}
$$

In particular, the mean of DITL distribution is

$$
\mu_{1}^{\prime}=\sum_{x=0}^{\infty} x\left[\frac{(1+2 x)^{\delta}}{(1+x)^{2 \delta}}-\frac{(3+2 x)^{\delta}}{(2+x)^{2 \delta}}\right]
$$

The central moments of DITL distribution can be obtained using the following relation

$$
\mu_{r}=\sum_{j=0}^{r}\left(\begin{array}{l}
r \\
j
\end{array}\right)(-1)^{j} \mu^{j} \mu_{r-j}^{\prime}
$$

The variance of DITL distribution is given as

$$
\operatorname{Var}(X)=\sum_{x=0}^{\infty} x^{2}\left[\frac{(1+2 x)^{\delta}}{(1+x)^{2 \delta}}-\frac{(3+2 x)^{\delta}}{(2+x)^{2 \delta}}\right]-\left\{\sum_{x=0}^{\infty} x\left[\frac{(1+2 x)^{\delta}}{(1+x)^{2 \delta}}-\frac{(3+2 x)^{\delta}}{(2+x)^{2 \delta}}\right]\right\}^{2}
$$

The dispersion index can be calculated using the expression

$$
D I=\frac{\text { Variance of DITD }}{\text { Mean of DITLD }}
$$

Since the above equation cannot be solved, so the descriptive measures, i.e., mean and variance are computed numerically. These measures are presented in Figure 3. 

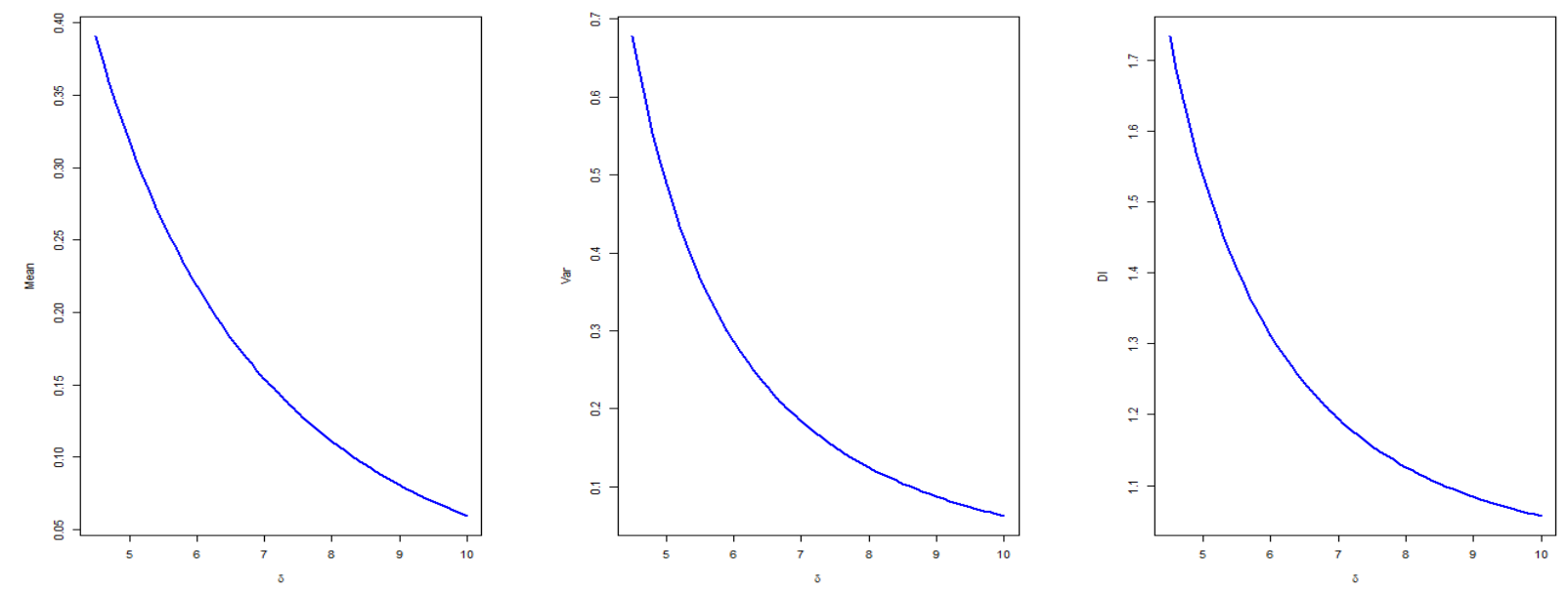

Figure 3: Plots of Mean, Variance and Dispersion Index for DITL distribution

From Figure 3, it is apparent that the mean, variance, and DI of the DITL distribution have decreasing behavior with an increase in parameter $\delta$.

\section{PARAMETER ESTIMATION OF DITL DISTRIBUTION}

This section describes the parameter estimation of DITL distribution using four different estimation methods. These methods are Maximum Likelihood Estimator (MLE), Cramer VonMises Estimator (CVM), Least Square Estimator (LS), and Weighted Least Square Estimator (WLSE).

\subsection{Method of Maximum Likelihood}

Let $x_{1}, x_{2}, x_{3}, \ldots, x_{n}$ be a random sample of size ' $n$ ' from DITL distribution with parameter $\delta$. The likelihood function is given by

$$
\begin{aligned}
L=\sum_{i=1}^{n} \log \left[\left(1+2 x_{i}\right)^{\delta}\left(2+x_{i}\right)^{2 \delta}-\left(3+2 x_{i}\right)^{\delta}\left(1+x_{i}\right)^{2 \delta}\right]-2 \delta \sum_{i=1}^{n} \log \left(1+x_{i}\right) \\
-2 \delta \sum_{i=1}^{n} \log \left(2+x_{i}\right)
\end{aligned}
$$

Now partially differentiate with respect to parameter $\delta$. 


$$
\begin{gathered}
\frac{\partial L}{\partial \delta}=\sum_{i=1}^{n} \frac{\left\{\begin{array}{c}
2\left(1+2 x_{i}\right)^{\delta}\left(2+x_{i}\right)^{2 \delta} \log \left(2+x_{i}\right)+\left(2+x_{i}\right)^{2 \delta}\left(1+2 x_{i}\right)^{\delta} \log \left(1+2 x_{i}\right) \\
-2\left(3+2 x_{i}\right)^{\delta}\left(1+x_{i}\right)^{2 \delta} \log \left(1+x_{i}\right)-\left(3+2 x_{i}\right)^{\delta} \log \left(3+2 x_{i}\right)\left(1+x_{i}\right)^{2 \delta}
\end{array}\right\}}{\left[\left(1+2 x_{i}\right)^{\delta}\left(2+x_{i}\right)^{2 \delta}-\left(3+2 x_{i}\right)^{\delta}\left(1+x_{i}\right)^{2 \delta}\right]} \\
-2 \sum_{i=1}^{n} \log \left(1+x_{i}\right)-2 \sum_{i=1}^{n} \log \left(2+x_{i}\right)
\end{gathered}
$$

The MLE of DITL distribution parameter $\delta$ can be obtained from the above Equation (11). The exact solution is not possible, so we can be obtained numerically.

\subsection{Method of Cramer von-Mises}

The Cramèr-von-Mises estimators (CVME), can be determined depending on the difference between both the estimated and exact distributions. The CVME estimator $\left(\hat{\delta}_{C V M}\right)$ can be obtained by minimizing

$$
\operatorname{CVM}(\delta)=\frac{1}{12 n}+\sum_{i=1}^{n}\left[F\left(x_{i}\right)-\frac{2_{i}-1}{2 n}\right]^{2}
$$

\subsection{Method of Least Squares and Weighted Least Squares}

The least-square estimator can be obtained by minimizing the sum of the square of residuals.

$$
L S(\delta)=\sum_{i=1}^{n}\left[F\left(x_{i}\right)-\frac{i}{n+1}\right]^{2}
$$

The weighted least squares estimators of the parameter of DITL distribution are obtained by minimizing

$$
W L S(\delta)=\sum_{i=1}^{n} \frac{(n+1)^{2}(n+2)}{n-i+1}\left[F\left(x_{i}\right)-\frac{i}{n+1}\right]^{2}
$$

with respect to parameter $\delta$.

\section{SIMULATION}

In this section, a simulation analysis evaluates the output of four different estimators of the DITL for different values of parameter $\delta$. We consider the different sample sizes $n=10,20,50$, and 100 for the different values of parameter $=(0.8,1,1.5,2,3,5,10)$. From DITL distribution, we generate 10000 iterations of random samples. For each computation, we get the average estimations (AEs) and mean square error (MSE). The output of considered estimators is compared in terms of MSE, with the lowest MSE values indicating the best successful technique of estimation. The R program is used to obtain simulation results. The AE and MSE values for the MLE, CVM, LS, and WLS 
approaches are shown in Table 1. Methods tend towards the true parameter values, suggesting that all estimators are asymptotically unbiased.

Table 1: The simulation results for the parameter $\delta$

\begin{tabular}{|c|c|c|c|c|c|c|c|c|c|}
\hline \multirow{2}{*}{$\begin{array}{c}\text { Para. } \\
\delta\end{array}$} & \multirow{2}{*}{ n } & \multicolumn{2}{|c|}{ MLE } & \multicolumn{2}{|c|}{ CVME } & \multicolumn{2}{|c|}{ LSE } & \multicolumn{2}{|c|}{ WSE } \\
\hline & & AEs & MSEs & AEs & MSEs & AEs & MSEs & AEs & MSES \\
\hline \multirow{4}{*}{0.8} & 10 & 0.9138 & 0.1310 & 1.0117 & 0.2847 & 0.9922 & 0.3008 & 0.9590 & 0.2406 \\
\hline & 20 & 0.8460 & 0.0376 & 0.9669 & 0.1519 & 0.9203 & 0.1012 & 0.9341 & 0.1144 \\
\hline & 50 & 0.8137 & 0.0142 & 0.9142 & 0.0476 & 0.9046 & 0.0414 & 0.8965 & 0.0380 \\
\hline & 100 & 0.8042 & 0.0065 & 0.8961 & 0.0224 & 0.8972 & 0.0249 & 0.8923 & 0.0210 \\
\hline \multirow{4}{*}{1.0} & 10 & 1.1199 & 0.1786 & 1.3289 & 0.5450 & 1.2527 & 0.4231 & 1.2878 & 0.5169 \\
\hline & 20 & 1.0729 & 0.0675 & 1.2515 & 0.2398 & 1.2063 & 0.2054 & 1.2225 & 0.2150 \\
\hline & 50 & 1.0200 & 0.0212 & 1.1863 & 0.0908 & 1.1841 & 0.0879 & 1.1623 & 0.0745 \\
\hline & 100 & 1.0113 & 0.0101 & 1.1653 & 0.0539 & 1.1742 & 0.0590 & 1.1414 & 0.0433 \\
\hline \multirow{4}{*}{1.5} & 10 & 1.6754 & 0.4174 & 2.0778 & 1.2522 & 1.9456 & 0.9305 & 1.9506 & 0.9876 \\
\hline & 20 & 1.5762 & 0.1524 & 1.9852 & 0.6515 & 1.9267 & 0.5357 & 1.8867 & 0.4822 \\
\hline & 50 & 1.5332 & 0.0543 & 1.9298 & 0.3412 & 1.9285 & 0.3425 & 1.8565 & 0.2574 \\
\hline & 100 & 1.5079 & 0.0213 & 1.9242 & 0.2546 & 1.8986 & 0.2330 & 1.8440 & 0.1781 \\
\hline \multirow{4}{*}{2.0} & 10 & 2.2598 & 0.7833 & 2.8469 & 2.1167 & 2.6438 & 1.5017 & 2.6569 & 1.6724 \\
\hline & 20 & 2.0934 & 0.2675 & 2.7375 & 1.2770 & 2.6655 & 1.0143 & 2.6394 & 1.0230 \\
\hline & 50 & 2.0488 & 0.1046 & 2.6844 & 0.7219 & 2.6551 & 0.6799 & 2.5891 & 0.5918 \\
\hline & 100 & 2.0202 & 0.0468 & 2.6759 & 0.5741 & 2.6522 & 0.5508 & 2.5782 & 0.4564 \\
\hline \multirow{4}{*}{3.0} & 10 & 3.6942 & 29.170 & 4.2539 & 3.9863 & 3.9756 & 2.8443 & 3.9646 & 3.1796 \\
\hline & 20 & 3.2014 & 0.7266 & 4.2718 & 2.8786 & 4.0647 & 2.2260 & 4.1605 & 2.7242 \\
\hline & 50 & 3.0637 & 0.2182 & 4.1455 & 1.7956 & 4.1121 & 1.7172 & 4.1168 & 1.7456 \\
\hline & 100 & 3.0366 & 0.1003 & 4.1571 & 1.5673 & 4.1206 & 1.4607 & 4.1275 & 1.5432 \\
\hline \multirow{4}{*}{5.0} & 10 & 11.356 & 497.89 & 6.6005 & 7.1286 & 5.7374 & 2.9089 & 6.0616 & 3.5941 \\
\hline & 20 & 5.7253 & 29.774 & 6.8668 & 6.2545 & 6.4529 & 4.2814 & 6.6718 & 4.9023 \\
\hline & 50 & 5.1582 & 0.7127 & 6.8237 & 4.3197 & 6.6485 & 3.6260 & 7.0444 & 5.4704 \\
\hline & 100 & 5.0605 & 0.3035 & 6.7486 & 3.5393 & 6.7070 & 3.3384 & 7.1014 & 5.0482 \\
\hline \multirow{4}{*}{10.0} & 10 & 51.192 & 3263.8 & 9.6885 & 0.9487 & 9.5835 & 1.5339 & 9.0152 & 2.6714 \\
\hline & 20 & 31.884 & 1671.7 & 9.8330 & 0.5029 & 9.6529 & 1.0747 & 9.7720 & 1.1258 \\
\hline & 50 & 15.195 & 355.64 & 9.9858 & 0.0241 & 9.9235 & 0.2293 & 11.7927 & 4.8997 \\
\hline & 100 & 10.609 & 24.082 & 9.9994 & 0.0081 & 9.9972 & 0.0099 & 12.7972 & 9.2633 \\
\hline
\end{tabular}


If $\delta=0.8,1,1.5$, and 2 , the MLE is the best estimation method in all sample sizes. If $\delta=3$ with sample size $n=10$, the LSE method is the best estimation method while in other sample sizes the MLE is the best method of estimation. If $\delta=5$ with sample sizes $n=10$ and 20 the LS is the best estimation method while the MLE is the best method in sample sizes $n=50$ and 100 . If $\delta=10$ the CVME is the best estimation method in all sample sizes.

\section{APPLICATION}

In this section, we illustrate the importance of the proposed distribution using two data sets. Both data sets are counts. Five one-parameter competitive distributions of the DITL distribution are Poison distribution, discrete Pareto distribution [13], discrete Rayleigh distribution [12], discrete inverse Rayleigh distribution [21], and discrete Burr-Hutke distribution [19].

The first data represents the number of deaths due to coronavirus in Pakistan. A sample of 44 deaths is considered from March 18, 2020, to April 30, 2020.

The second data set represents the number of deaths due to coronavirus in India. A sample of 51 deaths is considered from March 11, 2020, to April 30, 2020. Both data sets are plotted in Figure 4.
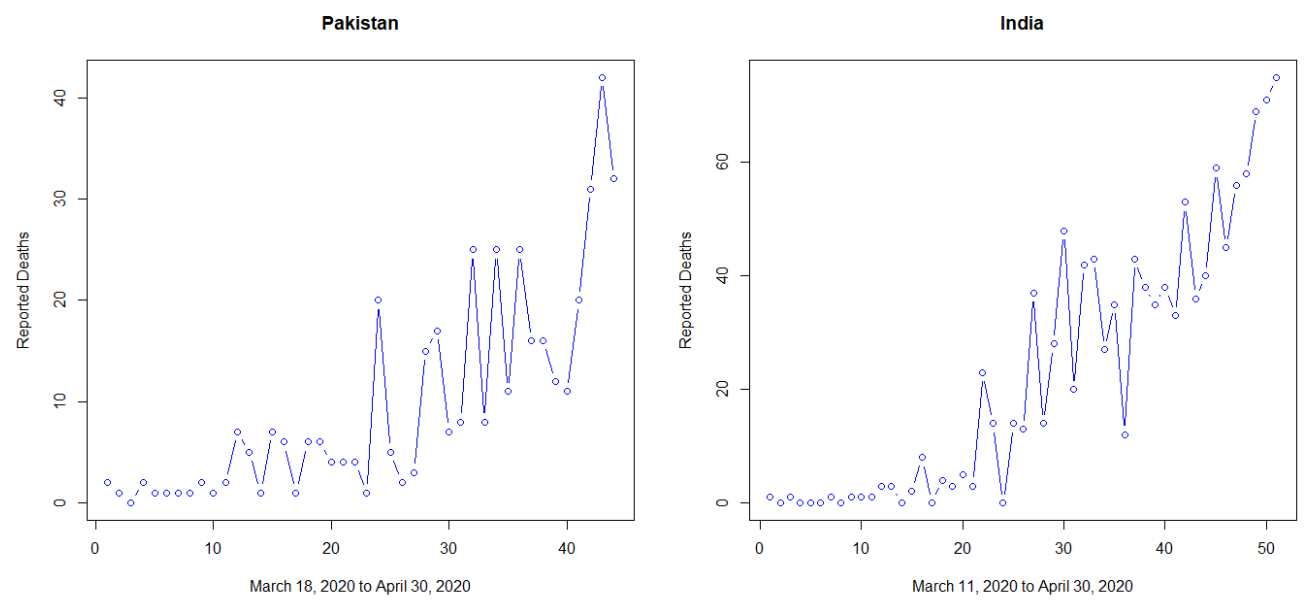

Figure 4. Plots for COVID-19 daily deaths in Pakistan and India

The results presented in Tables 2-3 and Figures 5-6 demonstrate the sufficiency and superiority of the proposed distribution in modeling the data sets when compared to the competitive distributions. 
Table 2: The MLEs, standard errors of the competing models for number of deaths in Pakistan

\begin{tabular}{lcccccc}
\hline \multirow{2}{*}{ Model } & \multicolumn{3}{c}{ MLE } & \multicolumn{3}{c}{ Goodness-of-fit Measures } \\
\cline { 2 - 7 } & $E(\delta)$ & SE & $\ell$ & AIC & BIC & KS \\
\hline DITLD & 0.70958 & 0.10705 & -153.04 & 308.09 & 309.87 & 0.1619 \\
\hline DPoi & 9.47694 & 0.46409 & -283.94 & 569.89 & 571.67 & 0.3910 \\
\hline DPr & 0.50220 & 0.07576 & -162.19 & 326.38 & 328.17 & 0.4010 \\
\hline DR & 9.98734 & 0.75335 & -168.85 & 339.70 & 341.49 & 0.3390 \\
\hline DIR & 7.43010 & 1.26260 & -166.31 & 334.61 & 336.40 & 0.3820 \\
\hline DBHD & 0.99483 & 0.01148 & -175.37 & 352.74 & 354.52 & 0.6470 \\
\hline
\end{tabular}

Table 3: The MLEs, standard errors of the competing models for number of deaths in India

\begin{tabular}{lcccccc}
\hline \multirow{2}{*}{ Model } & \multicolumn{2}{c}{ MLE } & \multicolumn{3}{c}{ Goodness-of-fit Measures } \\
\cline { 2 - 7 } & $E(\delta)$ & SE & $\ell$ & AIC & BIC & KS \\
\hline DITLD & 0.52980 & 0.07421 & -218.93 & 439.86 & 441.79 & 0.2190 \\
\hline DPoi & 22.6661 & 0.66665 & -740.90 & 1483.8 & 1485.7 & 0.4930 \\
\hline DPr & 0.40864 & 0.05726 & -221.91 & 445.82 & 447.75 & 0.2470 \\
\hline DR & 22.8734 & 1.60190 & -259.23 & 520.46 & 522.39 & 0.3780 \\
\hline DIR & 3.74690 & 0.59100 & -306.05 & 614.10 & 616.03 & 0.5470 \\
\hline DBHD & 0.99905 & 0.00443 & -249.99 & 501.97 & 503.91 & 0.5100 \\
\hline
\end{tabular}
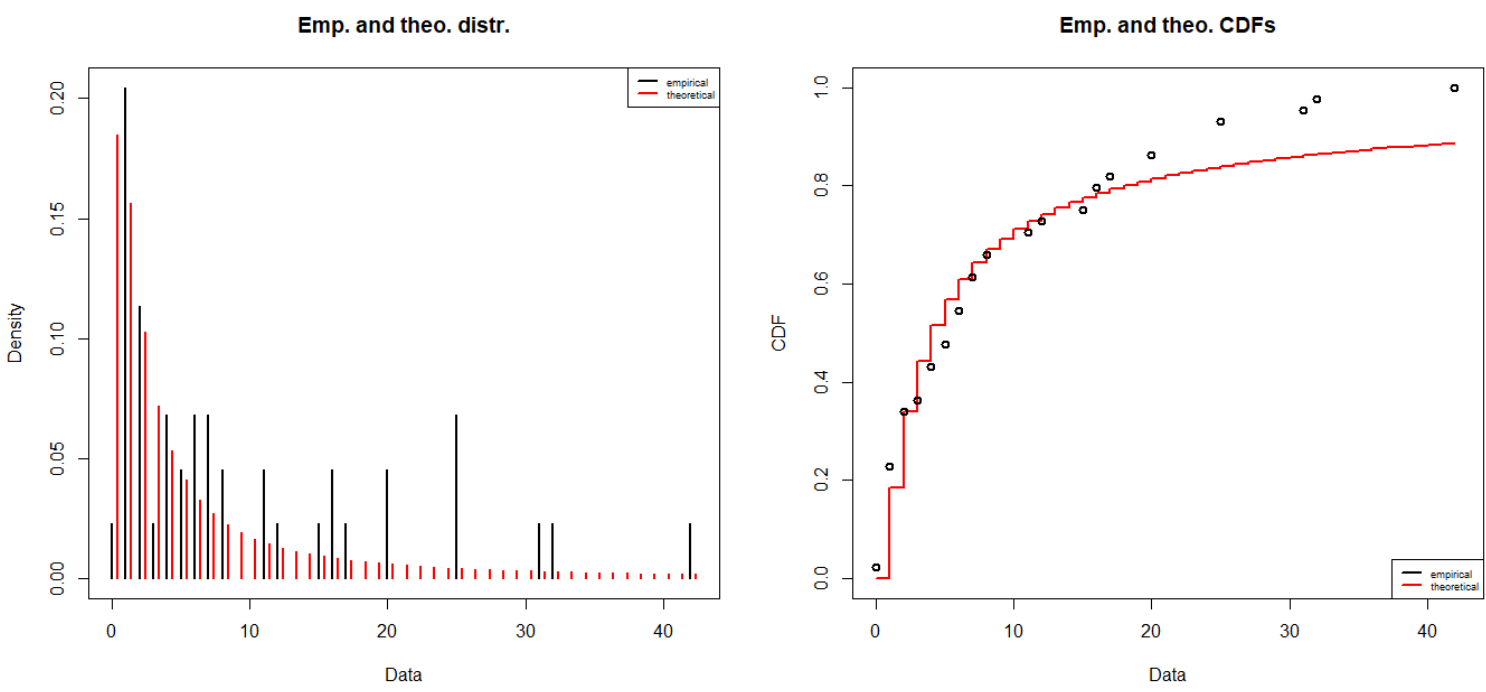

Figure 5: Density plot for the deaths due to COVID-19 in Pakistan 

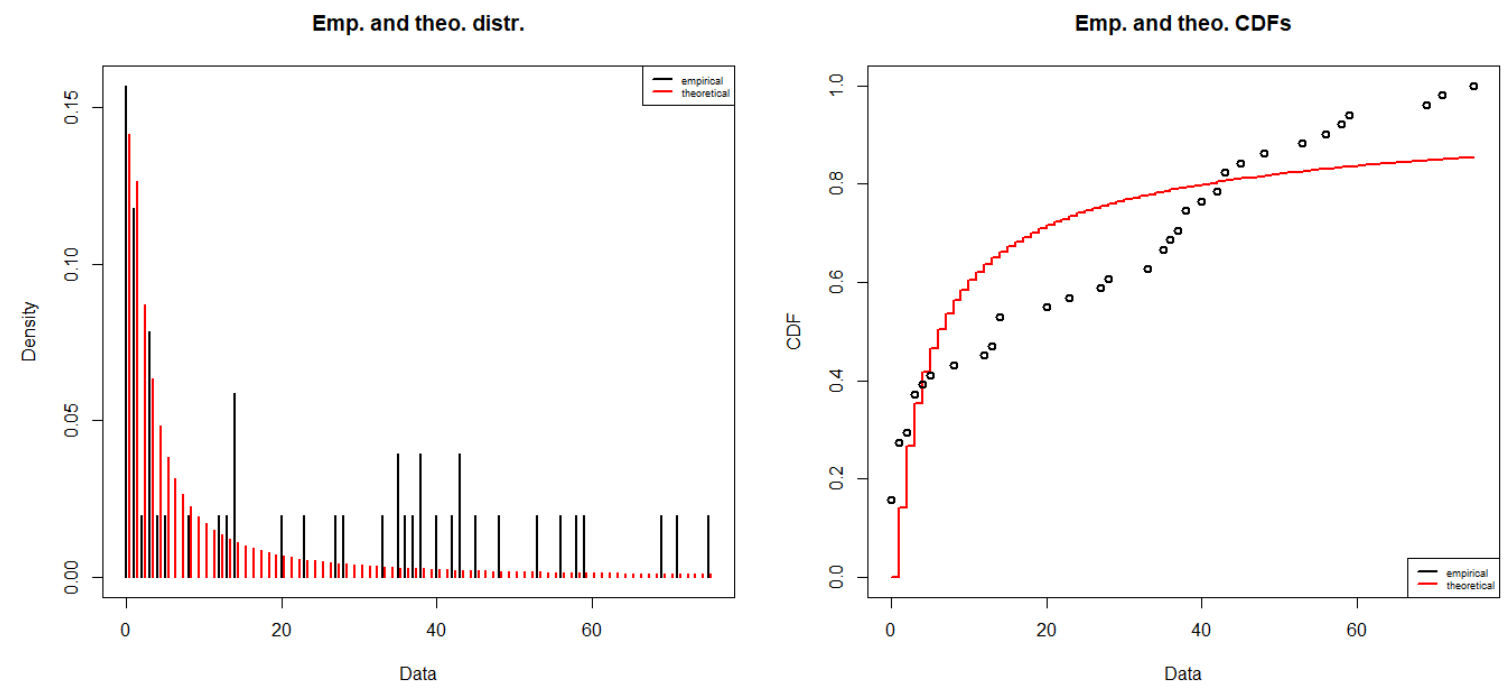

Figure 6: Density plot for the deaths due to COVID-19 in India

\section{CONCLUSION}

In this study, a new one-parameter discrete inverted Topp-Leone distribution has been proposed using a survival discretizing approach. Some structural properties of the proposed distribution are discussed. Different estimation methods including maximum likelihood estimation, leastsquare and weighted least squares estimation, and Cramer Von-Mises estimation have been presented. We carried out a simulation study to illustrate the performance of the parameter by different methods. Among all methods, the maximum likelihood estimator performs better for a large sample size. Application of the proposed DITL distribution in the analysis of two discrete data sets about Covid-19 has been presented. The DITL distribution, which has flexible features, is expected to make a significant contribution to the field of count data modeling.

Acknowledgement: Authors would like to express their gratitude to King Khalid University, Saudi Arabia for providing administrative and technical support.

Conflicts of Interest: The author(s) declare that there are no conflicts of interest regarding the publication of this paper. 


\section{REFERENCES}

[1] M. Ahsan-ul-Haq, M. Ahmed, J. Zafar, and P. L. Ramos, Modeling of COVID-19 Cases in Pakistan Using Lifetime, Ann. Data Sci. (2021), https://doi.org/10.1007/s40745-021-00338-9.

[2] A. A. Al-Babtain, Abdul Hadi N. Ahmed, and A. Z. Afify, A New Discrete Analog of the Continuous Lindley Distribution, with Reliability Applications, Entropy, 22(6) (2020), 603.

[3] E. M. Almetwally, H. M. Almongy and H. A. Saleh, Managing risk of spreading “COVID-19” in Egypt: Modelling using a discrete Marshall-Olkin generalized exponential distribution, Int. J. Probab. Stat. 9(2) (2020), 33-41.

[4] E. M. Almetwally, and G. M. Ibrahim, Discrete Alpha Power Inverse Lomax Distribution with Application of COVID-19 Data, Int. J. Appl. Math. 9(6) (2020), 11-22.

[5] B. Milan, Estimation of the Final Size of the Second Phase of the Coronavirus COVID 19 Epidemic by the Logistic Model. MedRxiv (Preprint), (2020b), https://doi.org/10.1101/2020.03.11.20024901.

[6] I. Nesteruk, Statistics Based Predictions of Coronavirus 2019-NCoV Spreading in Mainland China, MedRxiv, (2020), https:// doi.org/10.1101/2020.02.12.20021931.

[7] B. Milan, Estimation of the Final Size of the COVID-19 Epidemic. MedRxiv, (2020), https://doi.org/10.1101/2020.02.16.20023606.

[8] M. Maleki, M. R. Mahmoudi, D. Wraith, and K. Pho, Time series modelling to forecast the confirmed and recovered cases of COVID-19, Travel Med. Infect. Dis. 37 (2020), 101742.

[9] G. M. Ibrahim, and E. M. Almetwally, Discrete Marshall-Olkin Lomax Distribution Application of COVID-19, Biomed. J. Sci. Techn. Res. 32(5) (2021), 25381-25390.

[10]S. Chakraborty, Generating Discrete Analogues of Continuous Probability Distributions-A Survey of Methods and Constructions, J. Stat. Distrib. Appl. 2 (2015), 6.

[11]T. Nakagawa, and S. Osaki, The discrete Weibull distribution. IEEE Trans. Reliab. 24(5) (1975), 300-301.

[12] Roy, Dilip. Discrete Rayleigh distribution, IEEE Trans. Reliab. 53(2) (2004), 255-260.

[13] H. Krishna, and P. S. Pundir, Discrete Burr and Discrete Pareto Distributions, Stat. Methodol. 6(2) (2009), $177-88$.

[14] M. A. Jazi, D. L. Chin, and H. A. Mohammad, A Discrete Inverse Weibull Distribution and Estimation of Its Parameters. Stat. Methodol. 7(2) (2010), 121-32.

[15]E. Gómez-Déniz, and C. Enrique, The Discrete Lindley Distribution: Properties and Applications, J. Stat. Comput. Simul. 81(11) (2010), 1405-16.

[16] M. H. Alamatsaz, S. Dey, T. Dey and S. S. Harandi, Discrete Generalized Rayleigh Distribution, Pak. J. Stat. 32(1) (2016), 1-20. 
[17]E. Altun, M. El-Morshedy, and M. S. Eliwa, A Study on Discrete Bilal Distribution With Properties and Applications on integer-valued Autoregressive Process, Revstat. Stat. J. 18 (2020), 70-99.

[18] M. Shafqat, S. Ali, I. Shah and S. Dey, Univariate Discrete Nadarajah and Haghighi Distribution: Properties and Different Methods of Estimation, Statistica, 80(3) (2020), 301-330.

[19]M. El-Morshedy, M. S. Eliwa, and E. Altun, Discrete Burr-Hatke Distribution with Properties, Estimation Methods and Regression Model, IEEE Access, 8 (2020), 74359-70.

[20] A. S. Hassan, M. Elgarhy and R. Ragab, Statistical properties and estimation of inverted Topp-Leone distribution, J. Stat. Appl. Probab. 9(2) (2020), 319-331.

[21]T. Hussain, and M. Ahmad, Discrete Inverse Rayleigh Distribution, Pak. J. Stat. 30(2) (2014), 203-222. 